\title{
Structural and functional comparison of Saccharomonospora azurea strains in terms of primycin producing ability
}

\author{
Márk Kovács ${ }^{1,3}$. Dénes Seffer ${ }^{1}$. Ágota Pénzes-Hüvös ${ }^{1} \cdot$ Ákos Juhász $^{2} \cdot$ Ildikó Kerepesi $^{3} \cdot$ Kitti Csepregi $^{3}$. \\ Andrea Kovács-Valasek ${ }^{3} \cdot$ Csaba Fekete $^{3}$ (i)
}

Received: 16 July 2020 / Accepted: 15 September 2020 / Published online: 29 September 2020

(c) The Author(s) 2020

\begin{abstract}
Emerging and re-emerging microbial pathogens, together with their rapid evolution and adaptation against antibiotics, highlight the importance not only of screening for new antimicrobial agents, but also for deepening knowledge about existing antibiotics. Primycin is a large 36-membered non-polyene macrolide lactone exclusively produced by Saccharomonospora azurea. This study provides information about strain dependent primycin production ability in conjunction with the structural, functional and comparative genomic examinations. Comparison of high- and low-primycin producer strains, transcriptomic analysis identified a total of 686 differentially expressed genes (DEGs), classified into diverse Cluster of Orthologous Groups. Among them, genes related to fatty acid synthesis, self-resistance, regulation of secondary metabolism and agmatinase encoding gene responsible for catalyze conversion between guanidino/amino forms of primycin were discussed. Based on in silico data mining methods, we were able to identify DEGs whose altered expression provide a good starting point for the optimization of fermentation processes, in order to perform targeted strain improvement and rational drug design.
\end{abstract}

Keywords Polyketide biosynthetic genes $\cdot$ Primycin $\cdot$ Saccharomonospora azurea $\cdot$ Structural and functional genomics

\section{Introduction}

Actinomycetales are known as natural producers of a wide range of biologically active secondary metabolites that exhibit remarkable clinical importance (Jakubiec-Krzesniak et al. 2018; Solecka et al. 2012). Primycin, produced by a Gram-positive (G+) filamentous bacteria Saccharomonospora azurea, is a 36-membered marginolactone antibiotic that possesses high antimicrobial activity against frequent Gram-positive pathogens, including clinically prevalent

Electronic supplementary material The online version of this article (https://doi.org/10.1007/s11274-020-02935-x) contains supplementary material, which is available to authorized users.

Csaba Fekete

fekete@gamma.ttk.pte.hu

1 PannonPharma Pharmaceutical Ltd., 7720 Pécsvárad, Hungary

2 Faculty of Agricultural and Environmental Sciences, Institute of Biological Sciences, Szent István University, 2100 Gödöllő, Hungary

3 Faculty of Sciences, Institute of Biology, University of Pécs, 7624 Pécs, Hungary multidrug-resistant strains (Feiszt et al. 2014). In the last decade complete genome sequences have been obtained for several species of Saccharomonospora genus, however the available genomics information regarding biologically active metabolite production is still underrepresentated compared to other members of Pseudonocardiaceae family. Primycin was first described in 1954 to be produced by Streptomyces primycini, and thereafter by Micromonospora galeriensis, but none of these species was validly published (Szabó et al. 1976; Vályi-Nagy et al. 1954). In recent times, only S. azurea is known to has the ability to produce primycin (Csepregi et al. 2012; Klenk et al. 2012).

It is well documented that several clinically important bioactive secondary metabolites are synthesized on modular polyketide synthase (PKS) and non-ribosomal peptide synthase (NRPS) enzyme complexes via a conserved thiotemplate mechanism (Du and Lou 2010; Wang et al. 2014). Among them, macrolide antibiotics, represented by polyene and non-polyene compounds are one of the most effective antimicrobial agents. Primycin, like other guanidine-containing macrocyclic polyketides is also synthesized by the bacterial modular type I polyketide synthase pathway. In general, each PKS module minimally consists of a 
ketosynthase (KS), acyltransferase (AT), and an acyl carrier protein (ACP) catalytic domains, usually extended by ketoreductase (KR), dehydratase (DH), enolyreductase (ER) and thioesterase (TE) accessory domains. The 4-guanidinobutanoyl-COA starter unit of the primycin biosynthesis is derived from the three-step L-arginine precursor pathway, catalyzed by amine oxidase, amidohydrolase and acyl-CoA ligase enzymes (Hong et al. 2013). The activated 4-guanidinobutanoyl group is transferred to the loading ACP domain, performed by ACP S-malonyltransferase and subsequently the growing polyketide chain synthesis follows the canonical type I PKS biosynthetic logic. Finally, the TE domain catalyzes the release of the ACP-bound polyketide product through hydrolysis of the thioester bound. Although the modular genetic architecture of type I PKS is intensively reviewed (Hertweck 2009; Tsai and Ames 2009), knowledge about genetic factors controlling the yield and quality of secondary metabolites synthesis is limited.

The detailed genetic map of the extended primycin PKS gene cluster revealed the presence of glycosyltransferase and agmatinase encoding genes directly adjacent to the PKS. In the late step of primycin biosynthesis, transfer of the arabinosyl moiety onto the hydroxyl group of A1, A2 and $\mathrm{A} 3$ isoforms is catalysed by glycosyltransferase analogue to ECO-0501 biosynthesis pathway (Banskota et al. 2006). Agmatinase enzyme, as a member of the ureohydrolase superfamily, is responsible for catalyzing conversion between guanidino and amino forms of primycin, by a nucleophilic attack on the amidino carbon. The importance of agmatinase in primycin-like amino/guanidino marginolactones biosynthesis was highlighted by Hong and coworkers (Hong et al. 2016).

Even though a comparative structural genomic approach can provide important knowledge related to antibiotic gene clusters, it can not tell the whole story. To gain more insight into structure-function relationships beyond static analysis of biosynthetic gene cluster, the application of acombined approach of structural and functional genomics revealed several differentially expressed genes (DEGs), possibly involved in primycin biosynthetic pathway. Based on Cluster of Orthologous Groups (COG) and Gene Ontology (GO) systems, DEGs responsible for signal transduction, fatty acid synthesis and multidrug transport were emphasized.

\section{Materials and methods}

\section{Bacterial strains and culture conditions}

S. azurea DSM 44631, S. viridis DSM 43017, S. glauca DSM 43769 and S. cyanea DSM 44106 strains used in this study were purchased from Leibniz Institute, DSMZGerman Collection of Microorganisms and Cell Cultures, while S. azurea SZMC 14600 was originated from Szeged Microbiology Collection (SZMC). Bacterial cultures were stored as a suspension in Luria Bertani (LB) broth with $20 \%$ (v/v) glycerol at $-80^{\circ} \mathrm{C}$. Culture conditions were carried out according to Valasek et al. (2016). Briefly $1 \mathrm{~mL}$ bacterial cell suspension was inoculated into $50 \mathrm{~mL}$ seed medium containing 3\% (w/v) soy flour, $4.2 \%(\mathrm{w} / \mathrm{v})$ water soluble starch, $0.36 \%(\mathrm{w} / \mathrm{v}) \mathrm{NaCl}, 0.6 \%(\mathrm{w} / \mathrm{v}) \mathrm{CaCO}_{3}, 0.5 \%(\mathrm{w} / \mathrm{v})$ sunflower oil, $\mathrm{pH} 8.0$, and incubated for 2 days at $37^{\circ} \mathrm{C}$ in an orbital shaker at $200 \mathrm{rpm}$. Subsequently, $1 \mathrm{~mL}$ of accurately homogenized seed culture was transferred into $35 \mathrm{~mL}$ of fermentation medium containing $4 \%(\mathrm{w} / \mathrm{v})$ soy flour, $4 \%(\mathrm{w} / \mathrm{v})$ water soluble starch, $0.3 \%$ (w/v) $\mathrm{NaCl}, 0.5 \%$ (w/v) $\mathrm{CaCO}_{3}$, $0.3 \%(\mathrm{w} / \mathrm{v})$ stearic acid, $0.1 \%(\mathrm{w} / \mathrm{v}) \mathrm{KH}_{2} \mathrm{PO}_{4}, 0.6 \%(\mathrm{w} / \mathrm{v})$ sunflower oil ( $\mathrm{pH} 9.5$ ), and cultivated for 7 days at $28^{\circ} \mathrm{C}$ in an orbital shaker at $200 \mathrm{rpm}$. Bacillus subtilis ATCC 6633 used for agar well diffusion assay was purchased from American Type Culture Collection (ATCC).

\section{Antimicrobial assay}

Antimicrobial activity of n-butanol-ethanol-distilled water 1:1:2 (v/v) (BEW) extracts of 5 day fermented cells were determined by agar well diffusion assay (Balouiri et al. 2016). Accordingly, Bacillus subtilis ATCC 6633 test strain (approx. $10^{6} \mathrm{CFU} / \mathrm{mL}$ ) was inoculated into liquid phase $\left(50{ }^{\circ} \mathrm{C}\right)$ Mueller-Hinton agar (Biolab). After solidification of culture media, sample holding wells were prepared $(9 \mathrm{~mm}$ diameter, $5 \mathrm{~mm}$ depth). Original extracts were diluted ten times with BEW, and a subsequently twofold dilution series was prepared from each sample in ethanol-phosphate buffer (1.16\% (w/v) $\mathrm{K}_{2} \mathrm{HPO}_{4} ; 0.91 \%(\mathrm{w} / \mathrm{v}) \mathrm{KH}_{2} \mathrm{PO}_{4} ; 25 \%(\mathrm{v} / \mathrm{v})$ ethanol; $75 \%$ distilled water $(\mathrm{v} / \mathrm{v}))$. As a reference, standard crystallized primycin-sulphate stock solution $(1 \mathrm{mg} / \mathrm{mL}$ in BEW) was diluted ten times in BEW, and subsequent dilution steps were performed in ethanol-phosphate buffer to get $100 \mu \mathrm{g} / \mathrm{mL} ; 50 \mu \mathrm{g} / \mathrm{mL} ; 25 \mu \mathrm{g} / \mathrm{mL} ; 12.5 \mu \mathrm{g} / \mathrm{mL} ; 6.25 \mu \mathrm{g} / \mathrm{mL}$; $3.125 \mu \mathrm{g} / \mathrm{mL} ; 1.56 \mu \mathrm{g} / \mathrm{mL} ; 0.78 \mu \mathrm{g} / \mathrm{mL} ; 0.39 \mu \mathrm{g} / \mathrm{mL}$ final concentrations of primycin. Each plate contained control wells for $100 \mu \mathrm{L}$ known concentration of primycin-sulphate standard, as well as the same volume of sample extracts. Antibacterial activity was determined by the size of the inhibition zones after $20 \mathrm{~h}$ incubation at $30{ }^{\circ} \mathrm{C}$. Primycin concentrations of extracts were calculated according to the calibration curve fitted trend line equation. Samples were obtained from three independent fermentations and triplicated as technical replicates.

\section{Chromatographic analysis}

High performance liquid chromatography (HPLC) with diode array detection (DAD) and electrospray-mass spectrometry (ESI-MS) detection based analysis of $S$. azurea cell 
extracts were carried out according to Kovács et al. (2019). Primycin concentrations were determined from $0.5 \mathrm{~mL}$ fermentation media, collected between the third and seventh days of fermentation. All samples were prepared in three independent biological replicates and measured in technical triplicates. Statistical analysis was completed using oneway analyses of variance (ANOVA). Values are reported as mean $\pm \mathrm{SD}$ (standard deviation) and results were expected statistically significant when $\mathrm{p}<0.05$.

\section{Comparative genomics tools}

Genome sequencing methods of S. azurea SZMC 14600 have been described previously (Csepregi et al. 2012). Comparative genome analysis was carried out within the Integrated Microbial Genomes Database Expert Review (IMG-ER) system (https://img.jgi.doe.gov/cgi-bin/w/main. cgi) (Markowitz et al. 2009, 2012). Records of the annotated genomes are displayed by the following accession numbers in GeneBank: S. azurea SZMC 14600-AHBX01000000; S. azurea DSM44631-AGIU02000000; S. viridis DSM43017-ABUM01000000; S. glauca DSM43769AGJI00000000; S. cyanea DSM44103-AHLY00000000. In silico DNA-DNA hybridization (DDH) values among Saccharomonospora species were calculated by using the Genome-To-Genome Distance Calculator (GGDC) web server (https://ggdc.dsmz.de) (Meier-Kolthoff et al. 2013). Distance values were determined by the recommended Formula 2 for incomplete draft genomes.

\section{Identification and in silico structural analysis of primycin PKS gene cluster}

The primycin type I PKS gene cluster was identified and analyzed by antiSMASH (Antibiotics \& Secondary Metabolite Analysis Shell) (Blin et al. 2019). Database searches for homologues genes and proteins were performed using the National Center for Biotechnology Information (NCBI) BLAST server (Altschul et al. 1990). Domain analysis and motif search were done by SMART (Simple Modular Architecture Research Tool) (Letunic et al. 2012), SBSPKS (Structure Based Sequence Analysis of Polyketide Synthases) (Anand et al. 2010) and MEME (Multiple Em for Motif Elicitation) (Bailey et al. 2009). Multiple sequences alignment were performed by CLUSTAL W (Larkin et al. 2007).

\section{Transcriptomic analysis}

Total RNA was extracted from $50 \mathrm{mg}$ of cell paste collected from fermentation medium after five days according to the method described by Stiekema et al. (1988). After DNase treatment (Promega RQ1-RNase-free DNase) RNA quantity was measured by Qubit 2.0 Fluorometer (Thermo Fisher Scientific), and quality was determined by Agilent Bioanalyzer 2100 instrument (Agilent RNA 6000 Nano reagent kit). High quality total RNA samples (RIN > 8.5) from pooled biological replicates were processed using the SOLiD total RNA-Seq Kit (Thermo Fisher Scientific) according to the manufacturer's recommendation. Briefly $5 \mu \mathrm{g}$ of pooled RNA was enriched by depleting ribosomal RNA using RiboMinus rRNA Removal Kit (Life Technologies), and the leftover was fragmented using RNaseIII. Following enzymatic fragmentation the $100-200 \mathrm{bp}$ size fraction was selected and ligated with adaptors. The templates were reverse transcribed using ArrayScript Reverse Transcriptase. The cDNA library was purified using Qiagen MinElute PCR Purification Kit (Qiagen) and size-selected on a $6 \%$ TBE-Urea denaturing polyacrylamide gel. The 150-250 bp cDNA fraction was amplified using AmpliTaq polymerase, and purified by Agencourt AmPureXP Beads (Beckman Coulter). The concentration of each library was determined using the SOLiD Library TaqMan Quantitation Kit (Life Technologies), and clonally amplified on SOLiD P1 DNA Beads by emulsion PCR. Beads were deposited onto sequencing slides and sequenced on SOLiD V4 Instrument using 50-base sequencing chemistry.

\section{Bioinformatic analysis}

RNA-Seq data was analyzed using Galaxy's open source, web-based platform (https://usegalaxy.org) (Afgan et al. 2018). Reads of the whole transcriptome of S. azurea SZMC 14600 and $S$. azurea DSM 44631 were aligned to $S$. azurea SZMC 14600 genome using Bowtie2 alignment protocol. Transcriptome assembly and differential expression analysis were performed according to the Cufflinks RNASeq workflow (Trapnell et al. 2010). DEGs represented at least twofold change ( $>2$ or $<-2$ in $\log _{2}$ ) were functionally annotated using Blast $2 \mathrm{GO}$ version 5.2.4. software (Götz et al. 2008). To determine GO terms, functional annotation of each transcript was performed against the non-redundant (nr) protein database compiled by National Center for Biotechnology Information (NCBI) using BLASTx with $10^{\mathrm{e}-3}$ e-value threshold. The set of DEGs were classified into COG categories based on the Joint Genome Institute (JGI) Integrated Microbial Genomes \& Microbiomes (IGM/M) system.

\section{Real-time quantitative PCR analysis}

The expression of the agmatinase encoding gene was measured by quantitative real-time PCR (qRT-PCR) by ABI Prism 7900 Sequence Detection System (Applied Biosystems). Total RNA obtained from three independent fermentation processes were isolated using Quick-RNA MiniPrep 
Kit (Zymo Research), and quantified by Qubit 2.0 Fluorometer (Thermo Fisher Scientific). Reverse transcription was performed by RevertAid Reverse Transcriptase (Thermo Fisher Scientific) applying $1 \mu \mathrm{g}$ of total RNA according to the manufacturer's instructions. Agmatinase encoding cDNA was amplified using gene specific primers (forward: 5'-GTT GAACAGATACCGCTCGTC-3' and reverse: 5'-TGTCTC ACTCCTGAAGACCTC-3') and Ct values were detected by SYBR Green/ROX fluorescence chemistry (Thermo Fisher Scientific) in $25 \mu \mathrm{L}$ final volume. The thermal profile was as follows: $95^{\circ} \mathrm{C}$ for $10 \mathrm{~min}$ initial denaturation, followed by 40 cycles of $95^{\circ} \mathrm{C}$ for $15 \mathrm{~s}, 60^{\circ} \mathrm{C}$ for $30 \mathrm{~s} 72{ }^{\circ} \mathrm{C}$ for $30 \mathrm{~s}$ and a final dissociation step at $95{ }^{\circ} \mathrm{C}$ for $15 \mathrm{~s}, 60^{\circ} \mathrm{C}$ for $15 \mathrm{~s}$ and $95{ }^{\circ} \mathrm{C}$ for $15 \mathrm{~s}$. The relative gene expression was determined by using the $\Delta \Delta \mathrm{Ct}$ method, relative to glyceraldehyde 3-phosphate dehydrogenase (GAPDH) (forward: 5'-CTACACGCAGGACCAGAACC-3' and reverse: 5'-GTT CAGTTCGGGCAGGACGA-3') as an endogenous control. Each biological sample was measured in at least three technical replicates.

\section{Results}

\section{Primycin producing ability}

While $S$. viridis DSM 43017, S. glauca DSM 43769 and $S$. cyanea DSM 4410 strains can grow in the fermentation media, none of them were able to produce primycin, consequently no zone of inhibition was detected in the antimicrobial assay (Fig. 1). Each of the investigated S. azurea strains was primycin producer, nevertheless significant differences were observed in their production capacity. Based on agar well diffusion assay, calculated primycin concentration in case of $S$. azurea SZMC 14600 revealed approximately seven times more product $(1173 \pm 66.22 \mathrm{mg} / \mathrm{L})$ compared to $S$. azurea DSM $44631(168 \pm 9.35 \mathrm{mg} / \mathrm{L})$. Yields of primycin producing ability of $S$. azurea SZMC 14600 (highproducer) and DSM 44631 (low-producer) were confirmed by HPLC-DAD-ESI/MS analysis (Fig. 2) and summarized in Table 1.

\section{Comparative structural genomics}

Comparative whole genome analysis revealed a high percentage identity (93.4\%) within the HSPs (high-scoring segment pairs) between S. azurea SZMC 14600 and S. azurea DSM 44631, while in case of other species identity in HSPs ranged from 28.0 to $20.3 \%$ (Table 1S). Based on overall similarities to antibiotic producer $S$. azurea strains, $S$. cyanea, S. galuca and S. viridis were selected for detailed in silico structural analysis. In order to further improve reliability of gene presence/absence polymorphism calling, the distribution of COG functional categories were determined. Protein characterized in COG represented on average $61.7 \%$ of total gene number (Table $2 \mathrm{~S}$ ). Comparative analysis of identified COG categories revealed 1221 proteins commonly present in all compared species. The graphical
Fig. 1 Agar well diffusion assay of primycin extracts from various Saccharomonospora species. Numbers from 1 to 6 indicate serial dilutions of fermented cells extracts. Wells of crystallized primycin-sulphate standard correspond to the following concentrations: (1) $12.5 \mu \mathrm{g} / \mathrm{mL}$; (2) $6.25 \mu \mathrm{g} / \mathrm{mL}$; (3) $3.125 \mu \mathrm{g} / \mathrm{mL}$; (4) $1.56 \mu \mathrm{g} / \mathrm{mL}$;

(5) $0.78 \mu \mathrm{g} / \mathrm{mL}$; (6) $0.39 \mu \mathrm{g} / \mathrm{mL}$

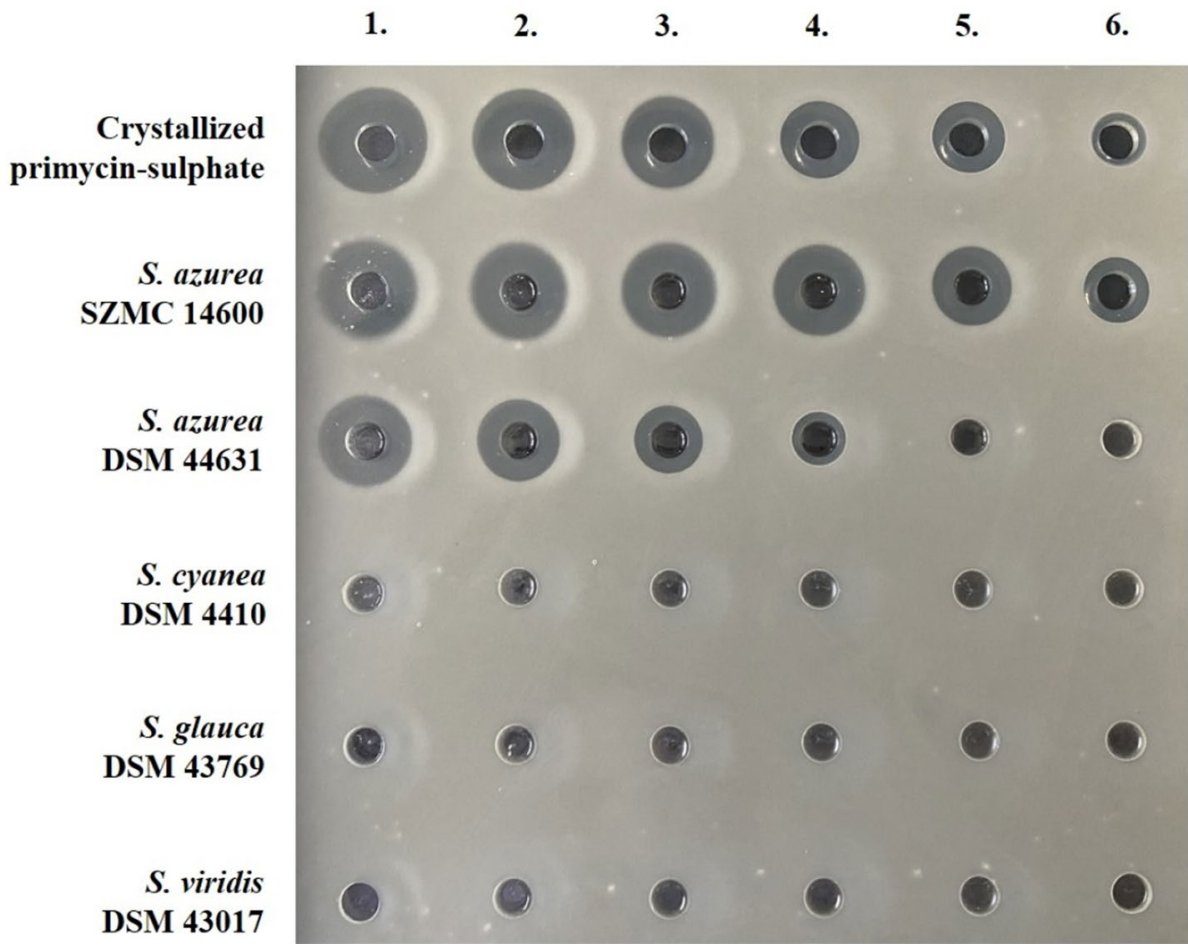



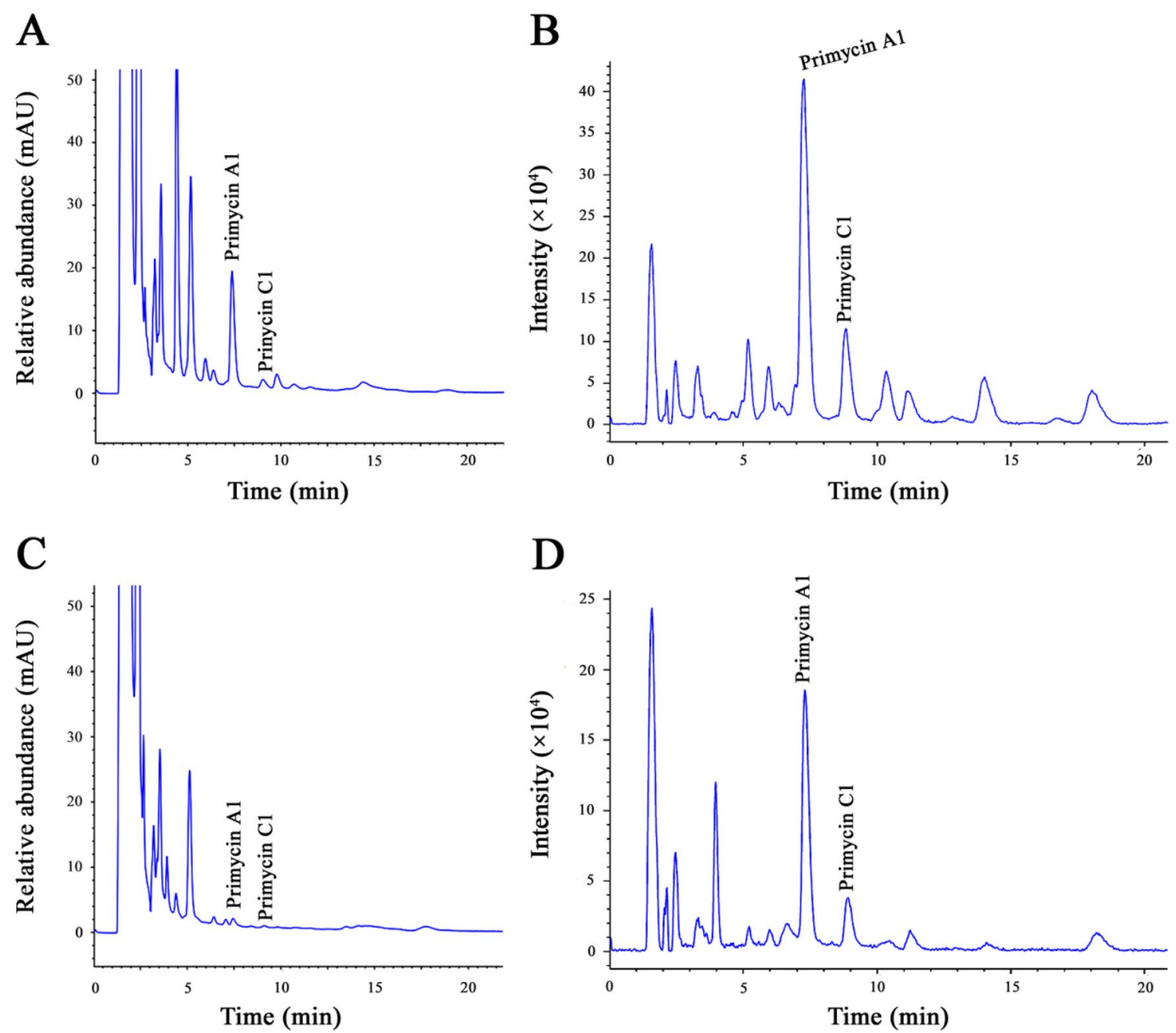

Fig. 2 Reversed-phase high-performance liquid chromatography (HPLC) and electrospray-mass spectrometry (ESI-MS) chromatograms of the high-primycin producer S. azurea SZMC 14600 (a, b) and low-primycin producer $S$. azurea DSM 44631 (c, d) cells extracts. HPLC chromatogram $(\mathbf{a}, \mathbf{c})$ peaks with retention time of

$7.38 \mathrm{~min}$ and $9.03 \mathrm{~min}$ correspond to primycin A1 (m/z 1078.7) and primycin $\mathrm{C} 1(\mathrm{~m} / \mathrm{z}$ 946.5) respectively, based on molecular masses detected by ESI-MS $(\mathbf{b}, \mathbf{d})$. Data was acquired for 22 min over a 950 to $1150 \mathrm{~m} / \mathrm{z}$ range in the positive ions mode

Table 1 Primycin yields of $S$. azurea SZMC 14600 and S. azurea DSM 44631 strains measured by high performance liquid chromatography (HPLC) with diode array detection (DAD) and electrospray-mass spectrometry (ESI-MS) detection at different fermentation stages

\begin{tabular}{llllll}
\hline Primycin concentration $(\mathrm{mg} / \mathrm{L})$ & & & \\
\hline Strain & Day 3 & Day 4 & Day 5 & Day 6 & Day 7 \\
\hline SZMC 14600 & $325.45 \pm 6.18$ & $728.24 \pm 21.65$ & $1113.89 \pm 36.10$ & $1248.08 \pm 44.37$ & $1261.06 \pm 15.54$ \\
DSM 44631 & $190.36 \pm 14.16$ & $220.10 \pm 8.68$ & $197.66 \pm 3.41$ & $219.81 \pm 6.45$ & $210.48 \pm 5.66$ \\
\hline
\end{tabular}

Concentration values $(\mathrm{mg} / \mathrm{L})$ are reported as means $\pm \mathrm{SD}$ of three independent experiment $(\mathrm{n}=3)$

representation of COGs (Fig. 3) displays 40 S. azurea specific proteins which were not present in any additional member of the comparison. Among them 5 proteins were unique for S. azurea SZMC 14600, and 14 proteins were present only for S. azurea DSM 44631. Further genome mining efforts on previously reported high quality draft genome data of S. azurea SZMC 14600 revealed complete primycin biosynthetic gene cluster (PBGC) consisting of type I PKS core genes flanked by accessory genes (Fig. 4), however in case of $S$. cyanea, $S$. galuca and $S$. viridis the antiSMASH 'in silico' genome analysis revealed the absence of primycin biosynthetic gene cluster. The predicted primycin PKS 


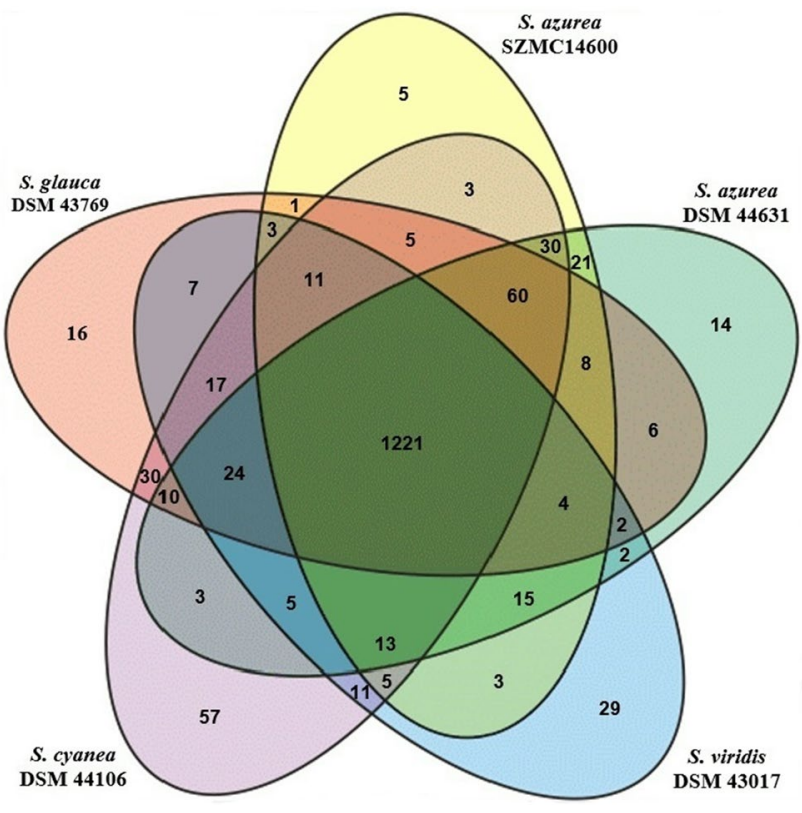

Fig. 3 Venn diagram representation of the distribution of Clusters of Orthologous Groups (COGs) in the five analyzed Saccharomonospora genomes. Numbers are indicating shared and unique genes annotated in COG database

model is composed of one loading and 18 separate extender modules, in good agreement with the chemical structure of primycin molecules (Fig. 5). The substrate specificity of PKS chain elongation is determined by AT domains. In case of primycin PKS, the amino acid sequence alignment of ATs revealed malonyl-CoA specific AT domains at module 4-10 and 12-16 having typical GHSx[LVIFAM] $\mathrm{G}$ and HAFH motifs (Fig. 1S). In modules 1-3, 11 and 17, methylmalonyl-CoA substrate specific motifs GHSx[QMI] $\mathrm{G}$ and YASH were observed (Fig. 2S) (Yadav et al. 2003; Zhang et al. 2019). Interestingly, antiSMASH in silicio analysis predicted unusual ethylmalonyl-CoA incorporation in module 18, however the presence of GHSQG and GAGH motifs define butylmalonyl-CoA, pentylmalonyl-CoA or hexylmalonyl-CoA incorporation (Fig. 3S).

\section{Transcriptomic analysis}

To gained more insight into difference at gene expression level between high- and low-primycin producer $S$. azurea strains RNA-Seq has been applied. High throughput ligation based SOLiD V4 sequencing revealed 48115904 and 33373930 trimmed reads corresponding to 484 and 336fold coverage of S. azurea SZMC 14600 and DSM 44631 genomes respectively. Following the Cufflinks RNA-Seq pipeline, transcriptomic analysis revealed 330 and 356 DEGs in a set of S. azurea SZMC 14600 vs. S. azurea DSM 44631 respectively at fold change cutoff of 2 in $\log _{2}$ scale. To identify functional categories of DEGs, GO enrichment analysis was performed. In case of high-primycin producer S. azurea SZMC 14600 strain, out of the 330 identified DEGs 253 were functionally annotated and classified into the three major GO categories as follows: 132 cellular component (C), 362 molecular function (F) and 231 biological process $(\mathrm{P})$. Among cellular components, the most frequent categories were integral components of membrane (GO:0016021), cytoplasm (GO:0005737), plasma membrane (GO:0005886), ATP-binding cassette (ABC) transporter complex (GO:0043190), and intracellular (GO:0005622). Regarding molecular functions, ATP binding (GO:0005524), DNA binding (GO:0003677), metal ion binding (GO:0046872), hydrolase activity (GO:0016787), transferase activity (GO:0016740), and methyltransferase activity (GO:0008168) were the most numerous categories. The top five biological component categories included oxidation-reduction process (GO:0055114), transmembrane transport (GO:0055085), regulation of transcription, DNAtemplated (GO:0006355), methylation (GO:0032259), and metabolic process (GO:0008152). In case of low-primycin producer strains, out of the 356 identified DEGs, 276 were functionally annotated and classified into: 133 cellular

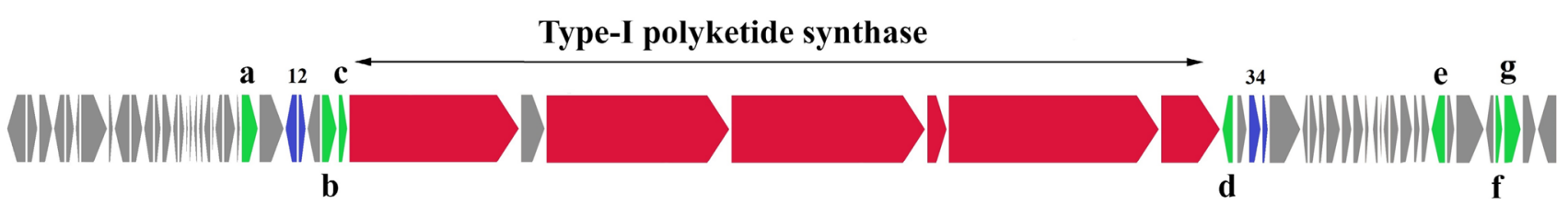

Fig. 4 Schematic genetic map of the primycin biosynthetic gene cluster. Genes labelled by a, b, c, d, e, f and $\mathrm{g}$ and marked by green representing acyl-CoA carboxylase (EHK80154.1); glycosyltransferase (EHK80161.1); S-malonyltransferase (EHK80162.1); agmatinase (EHK80172.1); acyl-CoA ligase (EHK88415.1); amidohydrolase (EHK88411.1) and amine oxidase (EHK88410.1) respectively. Type-I polyketide synthase core genes are represented by red arrows. Numbers from 1 to 4 above blue colored arrows indicate genes encoding $\mathrm{ABC}$ transporter ATP-binding protein (EHK80158.1); ABC-transporter transmembrane protein (EHK80159.1); two-component histidine kinase (EHK80176.1) and two-component system response regulator (EHK80177.1) respectively 


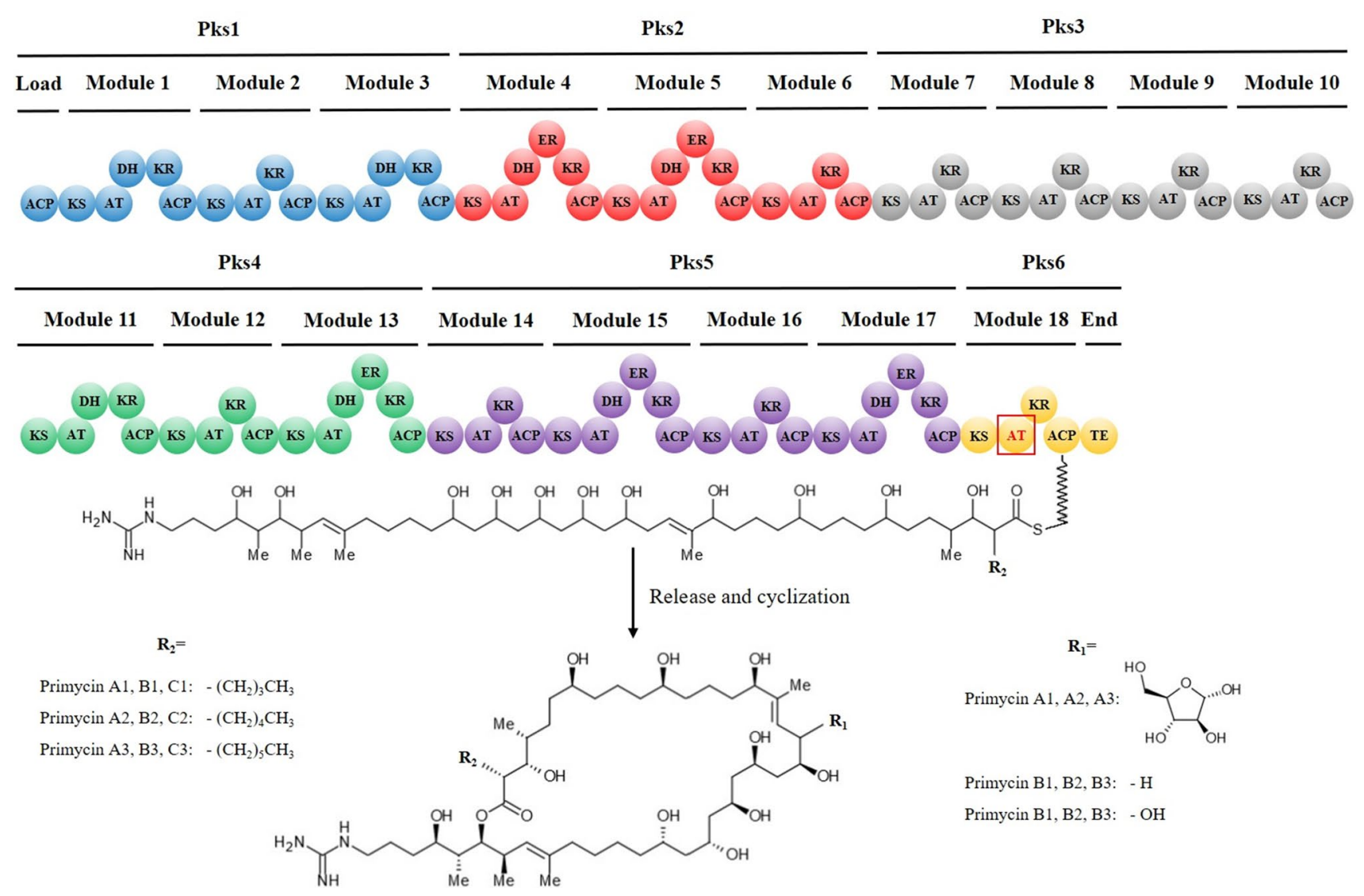

Fig. 5 Module and domain organisations of primycin type-I polyketide synthase and predicted polyketide chain as well as the assembled final product in Saccharomonospora azurea SZMC 14600. AT domain responsible for unusual extender unit incorporation, located in module 18 is marked by red and boxed. The major forms of primycin complex determined by $R_{1}$ and $R_{2}$ side chains is shown at the bottom of the figure

up- and down-regulation) as it is summarized in Table 3S. Taking into consideration the most characteristics expressional changes of high- and low-antibiotic producer strains, the following COG categories were remarkably down-regulated in SZMC 14600: carbohydrate transport and metabolism (G), cell wall/membrane/envelope biogenesis $(\mathrm{M})$, inorganic ion transport and metabolism $(\mathrm{P})$, and coenzyme transport and metabolism $(\mathrm{H})$. In contrast, the majority of DEGs belonging to COGs involved in nucleotide transport and metabolism (F), amino acid transport and metabolism (E), energy production and conversion (C) and defense mechanisms (V) were up-regulated in the high-producer strain (Fig. 6). The analysis of the primycin PKS core biosynthetic genes with respect to expression levels revealed no differences among the high- and lowprimycin producer $S$. azurea due to the two strains had comparable expression levels. As we presented in the schematic genetic map of the primycin gene cluster (Fig. 4), the agmatinase encoding gene (EHK80172.1) is located at the 3 ' end of the PKS. According to the RNA-Seq analysis, the agmatinase encoding gene belonging to the amino were also observed in a number of assigned genes (equal to 


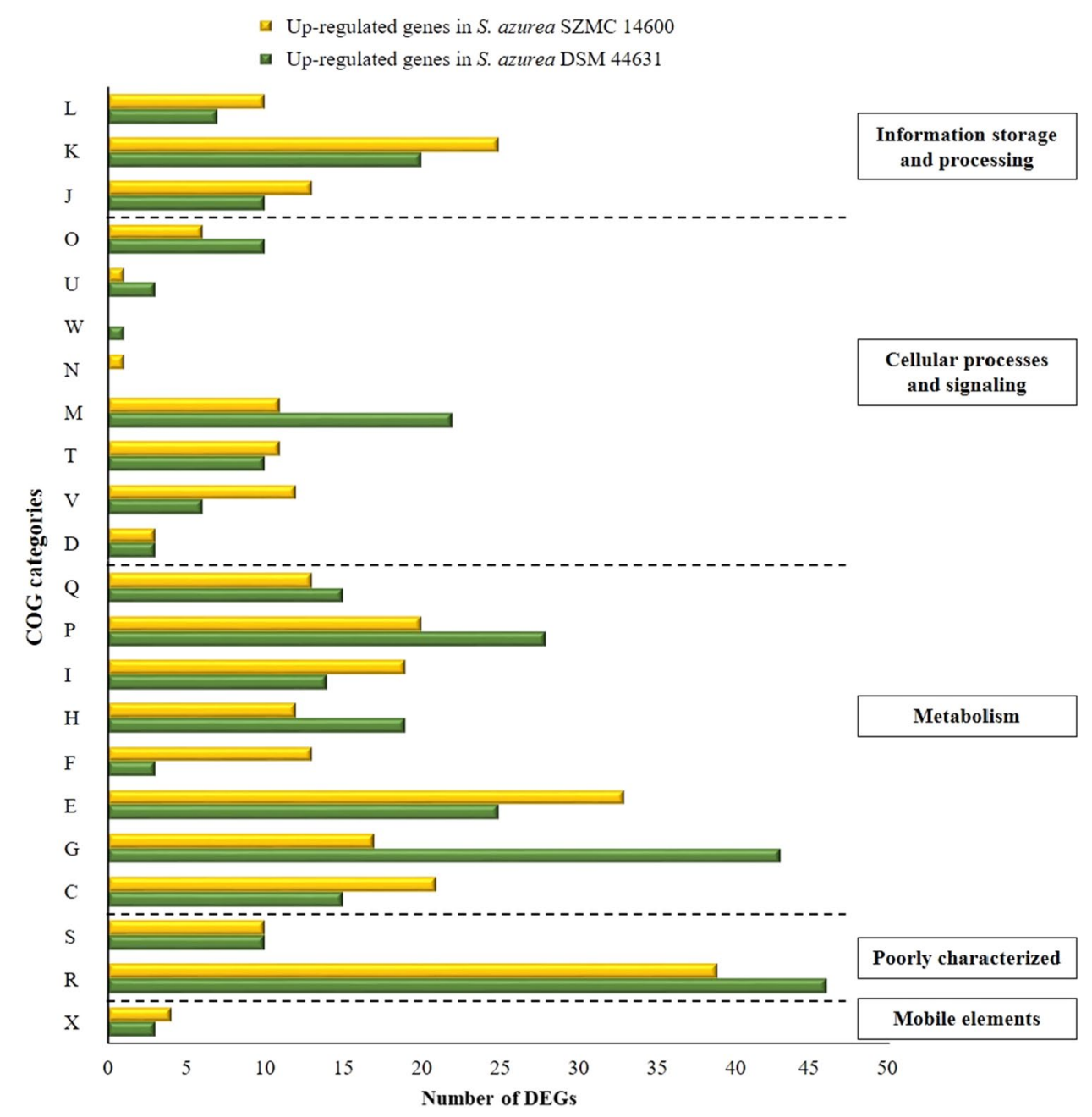

Fig. 6 Representation of Clusters of Orthologous Groups (COGs) functional categories of differentially expressed genes (DEGs) in $S$. azurea SZMC 14600 and S. azurea DSM 44631. Abbreviations of COG functional categories: (C) Energy production and conversion; (D) Cell cycle control, cell division, chromosome partitioning; (E) Amino acid transport and metabolism; (F) Nucleotide transport and metabolism; (G) Carbohydrate transport and metabolism; $(\mathrm{H})$ Coenzyme transport and metabolism; (I) Lipid transport and metabolism; (J) Translation, ribosomal structure and biogenesis; (K) Transcrip-

acid transport and metabolism (E) COG category was up-regulated in the primycin overproducer with $2.62 \log _{2}$ fold-change. The up-regulation tendency of agmatinase gene in S. azurea SZMC 14600 was confirmed by qRTPCR (Fig. 5S).

It is well known that polyketide and fatty acid (FA) synthesis are evolutionary closely related processes, and the two megasynthase assembly lines use homologous domains and share precursors such as acetyl- and malonyl-CoA (Smith and Tsai 2007). The expression profile of genes encoding 3-oxoacyl-(acyl-carrier-protein) synthases (EHK89245.1, EHK87608.1 and EHK84821.1) that play key roles regulating the product distribution of tion; (L) Replication, recombination and repair; (M) Cell wall/membrane/envelope biogenesis; (N) Cell motility; (O) Post-translational modification, protein turnover and chaperones; (P) Inorganic ion transport and metabolism; (Q) Secondary metabolites biosynthesis, transport and catabolism; (R) General function prediction only; (X) Mobilome: prophages, transposons; (S) Function unknown; (T) Signal transduction mechanisms; (U) Intracellular trafficking, secretion and vesicular transport; (V) Defense mechanisms; (W) Extracellular structures

FA synthesis were found to express lower levels in highprimycin producer strain.

Two ABC multidrug transporter encoding genes EHK80158.1 and EHK80159.1 located adjacent to PKS gene cluster (Fig. 4) that are linked to defense mechanisms (V) via COG were overexpressed in S. azurea SZMC 14600 with 8.0 and $3.8 \log _{2}$ fold-change respectively. Similarly, TetR family transcriptional regulators (TFRs), involved in control of a variety of processessuch as antibiotic production, efflux pump expression and multidrug and self-resistance-were also overexpressed in high-primycin producer strains within a range of 2.08 to $5.23 \log _{2}$ fold-change. 
Although comparative analysis of PBGC did not reveal structural differences between S. azurea SZMC 14600 and S. azurea DSM 44631, receptor histidine kinase (HK) and a cognate response regulator (RR) encoding genes (Fig. 4), elements of the two components' signal transduction system (TCS) were significantly up-regulated in high-primycin producer strain. An additional regulatory gene, encoding leucine-responsive regulatory protein (Lrp) was also differentially expressed between the two strains (Table 2).

\section{Discussion}

There is no doubt that antibiotic resistance is a global and serious issue nowadays (Payne et al. 2015). Even though a number of initiatives have been launched to reinvigorate the antibiotic research and development pipeline, an application of genome mining approach coupled with transcriptomic analysis and analytical methods is not yet common at industry level (Palazzotto and Weber 2018). On the basis of these innovative technologies, this study reports the result of a comparative structural and functional analysis of $S$. azurea strains in terms of primycin producing ability. Reexamination of primycin producing ability among representative species of the genus Saccharomonospora revealed that only $S$. azurea strains were capable of synthesizing primycin (Juhász et al. 2011), however the yield of antibiotic was significantly different according to agar well diffusion assay. Comparative HPLC-DAD-ESI/MS analysis of the two primycin producer S. azurea strains resulted in remarkable differences not only in yield, but also in dynamics of antibiotic production in each time points of batch fermentation. Enhancement of antibiotic producing ability of industrial strains remains the focus of industry driven research, nevertheless classical strain improvement and optimization of the fermentation process frequently suffers from limitations (Lal et al. 1996; Parekh et al. 2000). In order to assess the similarities and differences between $S$. azurea strains in regard to their potential to produce primycin, comparative whole genome analysis was performed. A wide range of biologically active natural products are synthesized by bacterial modular type I PKS assembly line (Hertweck 2015), such as 36-membered marginolactone primycin. Although our hypothesis, that structural differences of PKS gene clusters is responsible for elevated primycin production, was not supported by the comparative in silico analysis. Nevertheless, these efforts revealed the presence of unusual butlymalonyl-CoA, pentlymalonyl-CoA or hexylmalonyl-CoA substrate specificity in module 18. Similar AT domain characteristics were reported in case of stambomycin, thailandi, neoansamycin, antimycin and cinnabaramide biosynthesis (Greule et al. 2016; Li et al. 2015; Rachid et al. 2011; Ray et al. 2016). These findings were further supported by $R_{2}$ side chain variability of primycin molecules (Fig. 5). Regarding quantitative differences in
Table 2 Selected up- and downregulated genes represented by Clusters of Orthologous Groups (COGs) potentially related to primycin biosynthesis in comparison of high- and low-producer $S$. azurea strains, SZMC 14600 and DSM 44631 respectively

\begin{tabular}{|c|c|c|c|c|}
\hline \multirow[t]{2}{*}{ COG } & \multirow[t]{2}{*}{ Name } & \multirow[t]{2}{*}{ Accession no } & \multicolumn{2}{|c|}{ Relative fold-change $\left(\log _{2}\right)$} \\
\hline & & & $\begin{array}{l}\text { S. azurea } \\
\text { SZMC14600 }\end{array}$ & $\begin{array}{l}\text { S. azurea } \\
\text { DSM } 44631\end{array}$ \\
\hline \multicolumn{5}{|c|}{ Fatty acid synthesis } \\
\hline IQ & 3-oxoacyl-(acyl-carrier-protein) synthase & EHK89245.1 & - & 2.07 \\
\hline IQ & 3-oxoacyl-(acyl-carrier-protein) synthase & EHK87608.1 & - & 3.46 \\
\hline I & 3-oxoacyl-(acyl-carrier-protein) synthase III & EHK84821.1 & - & 2.17 \\
\hline \multicolumn{5}{|c|}{ Regulation of secondary metabolism } \\
\hline $\mathrm{T}$ & Two-component histidine kinase & EHK80176.1 & 2.57 & - \\
\hline KT & Two-component system response regulator & EHK80177.1 & 1.76 & - \\
\hline $\mathrm{K}$ & Lrp/AsnC family transcriptional regulator & EHK88701.1 & 4.24 & - \\
\hline \multicolumn{5}{|c|}{ Self-resistance } \\
\hline $\mathrm{V}$ & $\mathrm{ABC}$ transporter ATP-binding protein & ЕНК80158.1 & 8.01 & - \\
\hline $\mathrm{V}$ & ABC-transporter transmembrane protein & EHK80159.1 & 3.83 & - \\
\hline $\mathrm{K}$ & TetR/AcrR family transcriptional regulator & EHK82841.1 & 2.08 & - \\
\hline K & TetR/AcrR family transcriptional regulator & ЕНК88308.1 & 2.33 & - \\
\hline $\mathrm{K}$ & TetR/AcrR family transcriptional regulator & EHK82470.1 & 5.00 & - \\
\hline $\mathrm{K}$ & TetR/AcrR family transcriptional regulator & ЕНК88153.1 & 5.23 & - \\
\hline $\mathrm{K}$ & TetR/AcrR family transcriptional regulator & EHK88592.1 & 3.16 & - \\
\hline $\mathrm{K}$ & TetR/AcrR family transcriptional regulator & EHK83942.1 & 4.29 & - \\
\hline
\end{tabular}

Abbreviations of COG functional categories: (I) Lipid transport and metabolism; (K) Transcription; (Q) Secondary metabolites biosynthesis, transport and catabolism; (T) Signal transduction mechanisms; (V) Defense mechanisms 
primycin producing ability, much effort has been made in investigating complex gene expression profiles of $S$. azurea strains. RNA-Seq of high- and low-primycin producer $S$. azurea strains resulted 686 DEGs with cutoff 2 and classified into diverse COGs, however metabolism-related COG categories were dominant, representing more than $50 \%$ of the total hits (Table 3S). Among them, the agmatinase encoding gene belonging to amino acid transport and metabolism (E) was up-regulated 2.62-fold in the primycin overproducer strain. The relevance of agmatinase gene overexpression highlights its importance in conversion of amino/guanidino marginolactones biosynthesis, as mentioned in an earlier study (Hong 2016). More recently, a case study focused on targeting mechanisms of azalomycin $\mathrm{F}_{5 \mathrm{a}}$ produced by Streptomyces hygroscopicus var. azalomyceticus revealed that the guanidyl side chain of the marginolactone antibiotic plays pivotal role in antibacterial effect. Consequently, modification of agmatinase enzyme encoding gene expression emerges as a promising target to enhance antibacterial activity (Yuan et al. 2019). Expression profiling revealed DEGs encoding enzymes responsible for fatty acid biosynthesis. The enzymatic machinery responsible for de novo biosynthesis of FAs and polyketides possess many common features, including the utilization of identical precursors (Cronan and Thomas 2009), therefore substrate competition effect theoretically could not be excluded. Our results were based on the actual experimental design, in which high- and low-primycin producer $S$. azurea strains were compared under identical culture conditions, and growth phase did not support the hypothesis that competition generally does not occur due to the time shift of the two biosynthetic process (Gago et al. 2011). The overexpression of FA synthesis related genes in low-producer $S$. azurea DSM 44631 strain suggests that biosynthetic activity of FA and polyketide related machinery is not a clear sequential process, rather should be considered an overlapping even at least partially.

Primycin, a non-polyene marginolactone antibiotic produced by filamentous bacteria $S$. azurea, possesses high antimicrobial activity against frequent $\mathrm{G}+$ pathogens, including clinically prevalent multidrug-resistant strains (Feiszt et al. 2014). To protect themselves against their own bioactive metabolites, self-resistance mechanism for antibiotic producers are crucial. Despite the fact that the history of primycin dates back more than 60 years, there is no available scientific knowledge of the regulation of self-resistance in $S$. azurea species. Among members of the $\mathrm{ABC}$ transporter superfamily, encoding genes involved in multidrug and self-resistance (Rees et al. 2009; van Veen and Konings 1998), tightly linked to the PBGC were up-regulated in overproducer strain. Similarly, six genes encoding TetR family of regulators (TFRs), associated with antibiotic resistance (Cuthbertson and Nodwell 2013; Deng et al. 2013), were also significantly overexpressed.
These findings support the hypothesis that the expression of resistance genes is presumably induced by primycin or intermediate molecules in a concentration related manner.

A bacterial two component signal transduction system (TCS) is not only important for complex adaptive responses towards environmental changes, but also involved in the biosynthetic control of a broad range of secondary metabolites (Rodríguez et al. 2013; Zschiedrich et al. 2016). For instance the two component system AfsQ1/Q2 of Streptomyces coelicolor was found to be capable of stimulating actinorhodin, undecylprodigiosin and calcium-dependent antibiotic production (Wang et al. 2013). Furthermore, it was demonstrated that orf 22 and orf 23 members of Streptomyces clavuligerus TCS positively regulate clavulanic acid biosynthesis, and their overexpression resulted in elevated yield of antibiotic production (Jnawali et al. 2008). To the extent of our knowledge, this is the first report to demonstrate overexpression of $\mathrm{HK}$ and RR encoding genes related to elevated primycin biosynthetic capacity in S. azurea.

Transcriptional regulation of secondary metabolisms in Streptomyces has been extensively studied (Bibb 2005), however there is still room for improvement in case of industrially important rare Actinomycetes. Genes corresponding to Lrp/AsnC family transcriptional regulators were found substantially up-regulated in high-primycin producer strains. Present findings were in agreement with previously reported facts that, genes encoding the Lrp/AsnC family transcriptional regulators (SCO2140 and SCO3361) act as positive regulators of antibiotic production in S. coelicolor (Liu et al. 2017; Yu et al. 2016).

In summary, we have presented a comprehensive study based on multidisciplinary approaches e.g. traditional microbiology; analytical chemistry; structural, functional and comparative genomics supported by a wide variety of bioinformatics tools. The primary aim of the research was to gain insight into the difference and determinants of primycin producing ability via comparison of high- and low-antibiotic producer $S$. azurea strains. Even though an in silico analysis of PKS gene clusters did not revealed significant structural differences between the two strains, clear evidence was found for unusual substrate specificity of the AT domain in module 18. Concerning quantitative differences in primycin producing ability, the performed transcriptomic analysis resulted several DEGs, classified into various COG categories. Among them, genes related to fatty acid synthesis, self-resistance, regulation of secondary metabolism and an agmatinase encoding gene responsible for catalyze conversion between guanidino and amino forms of primycin were discussed. As a result our efforts to investigate PBGC and the regulation of primycin biosynthesis provides clues for antibiotic yield- and strain-improvement as well as laying the foundation for rational drug design. 
Acknowledgements This research was partly supported by GINOP2.1.2-8.1.4-16 and GINOP-2.3.2.-15-2016-00047 grants and by PannonPharma, Ltd., Pécsvárad, Hungary.

Funding Open access funding provided by University of Pécs.

\section{Compliance with ethical standards}

Conflict of interest The authors declare that they have no conflict of interest.

Ethical approval This article does not contain any studies with human participants or animals performed by any of the authors.

Open Access This article is licensed under a Creative Commons Attribution 4.0 International License, which permits use, sharing, adaptation, distribution and reproduction in any medium or format, as long as you give appropriate credit to the original author(s) and the source, provide a link to the Creative Commons licence, and indicate if changes were made. The images or other third party material in this article are included in the article's Creative Commons licence, unless indicated otherwise in a credit line to the material. If material is not included in the article's Creative Commons licence and your intended use is not permitted by statutory regulation or exceeds the permitted use, you will need to obtain permission directly from the copyright holder. To view a copy of this licence, visit http://creativecommons.org/licenses/by/4.0/.

\section{References}

Altschul SF, Gish W, Miller W, Myers EW, Lipman DJ (1990) Basic local alignment search tool. J Mol Biol 215:403-410

Anand S, Prasad MVR, Yadav G, Kumar N, Shehara J, Ansari MZ, Mohanty D (2010) SBSPKS: Structure based sequence analysis of polyketide synthases. Nucleic Acids Res 38:W487-496

Bailey TL, Boden M, Buske FA, Frith M, Grant CE, Clementi L, Ren J, Li WW, Noble WS (2009) MEME Suite: tools for motif discovery and searching. Nucleic Acids Res 37:W202-208

Balouiri M, Sadiki M, Ibnsouda SK (2016) Methods for in vitro evaluating antimicrobial activity: a review. J Pharm Anal 6:71-79

Banskota AH, McAlpine JB, Sørensen D, Ibrahim A, Aouidate M, Pira AAM, Farnet CM, Zazopoulos E (2006) Genomic analyses lead to novel secondary metabolites. Part 3. ECO-0501, a novel antibacterial of a new class. J Antibiot 59(9):533-542

Bibb MJ (2005) Regulation of secondary metabolism in streptomycetes. Curr Opin Microbiol 8:208-215

Blin K, Shaw S, Steinke K, Villebro R, Ziemert N, Lee SY, Medema MH, Weber T (2019) antiSMASH 5.0: updates to the secondary metabolite genome mining pipeline. Nucleic Acids Res 47:W81-W87

Cronan JE, Thomas J (2009) Bacterial fatty acid synthesis and its relationships with polyketide synthetic pathways. Methods Enzymol 459:395-433

Csepregi K, Valasek A, Pénzes Á, Zs T, Kiss ÍÉ, Kerepesi I, Horváth B, Nagy I, Cs F (2012) Draft genome sequence of an efficient antibiotic-producing industrial strain of Saccharomonospora azurea, SZMC 14600. J Bacteriol 194(5):1263

Cuthbertson L, Nodwell JR (2013) The TetR family of regulators. Microbiol Mol Biol R 77(3):440-475

Deng W, Li C, Xie J (2013) The underling mechanism of bacterial TetR/AcrR family transcriptional repressors. Cell Signal 25:1608-1613
Du L, Lou L (2010) PKS and NRPS release mechanisms. Nat Prod Rep 27:255-278

Feiszt P, Gy M, Kerényi M, Dobay O, Szabó J, Zs D, Urbán E, Emôdy L (2014) Re-evaluation of in vitro activity of primycin against prevalent multiresistant bacteria. Int J Med Microbiol 304:1077-1085

Gago G, Diacovich L, Arabolaza A, Tsai SC, Gramajo H (2011) Fatty acid biosynthesis in actinomycetes. FEMS Microbiol Rev 35:475-497

Götz S, García-Gómez J, Terol J, Williams T, Nagaraj S, Nueda M, Robles M, Talón M, Dopazo J, Conesa A (2008) High-throughput functional annotation and data mining with the Blast2GO suite. Nucleic Acids Res 36:3420-3435

Greule A, Intra B, Flemming S, Rommel MG, Panbangred W, Bechthold A (2016) The draft genome sequence of Actinokineospora bangkokensis 44EHWT reveals the biosynthetic pathway of the antifungal thailandin compounds with unusual butylmalonylCoA extender units. Molecules 21(11):1607

Hertweck C (2009) The biosynthetic logic of polyketide diversity. Angew Chem Int Ed 48:4688-4716

Hertweck C (2015) Decoding and reprogramming complex polyketide assembly lines: Prospects for synthetic biology. Trends Biochem Sci 40:189-199

Hong H, Fill T, Leadlay PF (2013) A common origin for guanidinobutanoate starter units in antifungal natural products. Angew Chem Int Edit 52:13096-13099

Hong H, Samborskyy M, Lindner F, Leadlay PF (2016) An amidinohydrolase provides the missing link in the biosynthesis of amino marginolactone antibiotics. Angew Chem Int Ed 55:1118-1123

Jakubiec-Krzesniak K, Rajnisz-Mateusiak A, Guspiel A, Ziemska J, Solecka J (2018) Secondary metabolites of actinomycetes and their antibacterial, antifungal and antiviral properties. Pol J Microbiol 67(3):259-272

Jnawali HN, Oh T, Liou K, Park BC (2008) Two-component regulatory system involved in clavulanic acid production. $\mathrm{J}$ Antibiot 61:651-659

Juhász Á, Pénzes Á, Péteri Z, Pallos JP, Seffer D, Feiszt P, Pesti M, Fekete Cs, Vágvölgyi Cs, Gazdag Z, Papp G (2011) Process for producing primycin, primycin component(s), precursors and metabolites thereof via fermentation by the use of bacterial species Saccharomonospora azurea. WIPO patent application WO/2011/051741

Klenk HP, Held B, Lucas S, Lapidus A, Copeland A, Hammon N, Pitluck S, Goodwin LA, Han C, Tapia R, Brambilla EM, Pötter G, Land M, Ivanova N, Rohde M, Göker M, Detter JC, Kyrpides NC, Woyke T (2012) Genome sequence of the soil bacterium Saccharomonospora azurea type strain (DSM 44631T). Stand Genomic Sci 6:220-229

Kovács M, Sefferné Szalai M, Seffer D, Pallos JP, Drávavölgyi G, Kovács-Valasek A, Kerepesi I (2019) Understanding the role of fatty acid substrates on primycin biosynthesis by Saccharomonospora azurea during batch fermentation. Nat Prod Commun 14:1-6

Lal R, Khanna R, Kaur H, Khanna M, Dhingra N, Lal S, Gartemann KH, Eichenlaub R, Ghosh PK (1996) Engineering antibiotic producers to overcome the limitations of classical strain improvement programs. Crit Rev Microbiol 22(4):201-255

Larkin MA, Blackshields G, Brown NP, Chenna R, McGettigan PA, McWilliam H, Valentin F, Wallace IM, Wilm A, Lopez R, Thompson JD, Gibson TJ, Higgins DG (2007) Clustal W and Clustal X version 2.0. Bioinformatics 23:2947-2948

Letunic I, Doerks T, Bork P (2012) SMART 7: recent updates to the protein domain annotation resource. Nucleic Acids Res 40:D302-305

Li S, Li Y, Lu C, Zhang J, Zhu J, Wang H, Shen Y (2015) Activating a cryptic ansamycin biosynthetic gene cluster to produce 
three new naphthalenic octaketide ansamycins with n-pentyl and n-butyl side chains. Org Lett 17:3706-3709

Liu J, Li J, Dong H, Chen Y, Wang Y, Wu H, Li C, Weaver D, Zhang L, Zhang B (2017) Characterization of an Lrp/AsnC family regulator SCO3361, controlling actinorhodin production and morphological development in Streptomyces coelicolor. Appl Microbiol Biot 101(14):5773-5783

Markowitz VM, Mavromatis K, Ivanova NN, Chen IM, Chu K, Kyrpides NC (2009) IMG ER: a system for microbial genome annotation expert review and curation. Bioinformatics 25:2271-2278

Markowitz VM, Chen IMA, Palaniappan K, Chu K, Szeto E, Grechkin Y, Ratner A, Jacob B, Huang J, Williams P, Huntemann M, Anderson I, Mavromatis K, Ivanova NN, Kyrpides NC (2012) IMG: the integrated microbial genomes database and comparative analysis system. Nucleic Acids Res 40:D115-122

Meier-Kolthoff JP, Auch AF, Klenk H-P, Oker MG (2013) Genome sequence-based species delimitation with confidence intervals and improved distance functions. BMC Bioinformatics 14:60

Palazzotto E, Weber T (2018) Omics and multi-omics approaches to study the biosynthesis of secondary metabolites in microorganisms. Curr Opin Microbiol 45:109-116

Parekh S, Vinci VA, Strobel RJ (2000) Improvement of microbial strains and fermentation processes. Appl Microbiol Biot 54:287-301

Payne DJ, Miller LF, Findlay D, Anderson J, Marks L (2015) Time for a change: addressing R\&D and commercialization challenges for antibacterials. Philos Trans R Soc B 370:20140086

Rachid S, Huo L, Herrmann J, Stadler M, Köpcke B, Bitzer J, Müller R (2011) Mining the cinnabaramide biosynthetic pathway to generate novel proteasome inhibitors. ChemBioChem 12:922-931

RamirezAfgan E, Baker D, Batut B, van den Beek M, Bouvier D, Čech M, Chilton J, Clements D, Coraor N, Grüning B, Guerler A, Hillman-Jackson J, Jalili V, Rasche H, Soranzo N, Goecks J, Taylor J, Nekrutenko A, Blankenberg D (2018) The Galaxy platform for accessible, reproducible and collaborative biomedical analyses: 2018 update. Nucleic Acids Res 46(W1):W537-W544

Ray L, Valentic TR, Miyazawa T, Withall DM, Song L, Milligan JC, Osada H, Takahashi S, Tsai SC, Challis GL (2016) A crotonylCoA reductase-carboxylase independent pathway for assembly of unusual alkylmalonyl-CoA polyketide synthase extender units. Nat Commun 7:13609

Rees DC, Johnson E, Lewinson O (2009) ABC transporters: the power to change. Nat Rev Mol Cell Biol 10(3):218-227

Rodríguez H, Rico S, Díaz M, Santamaría R (2013) Two-component systems in Streptomyces: key regulators of antibiotic complex pathways. Microb Cell Fact 12:127

Smith S, Tsai SC (2007) The type I fatty acid and polyketide synthases: a tale of two megasynthases. Nat Prod Rep 24:1041-1072

Solecka J, Zajko J, Postek M, Rajnisz A (2012) Biologically active secondary metabolites from Actinomycetes. Cent Eur J Biol 7(3):373-390
Stiekema WJ, Heidekamp F, Dirkse WG, van Beckum J, de Haan P, Bosch CT, Louwerse JD (1988) Molecular cloning and analysis of four potato tuber mRNAs. Plant Mol Biol 11:255-269

Szabó IM, Marton M, Kulcsár G, Buti I (1976) Taxonomy of primycin producing actinomycetes. I. Description of the type strain of Thermomonospora galeriensis. Acta Microbiol Acad Sci Hung 23(4):371-376

Trapnell C, Williams BA, Pertea G, Mortazavi AM, Kwan G, van Baren MJ, Salzberg SL, Wold B, Pachter L (2010) Transcript assembly and abundance estimation from RNA-Seq reveals thousands of new transcripts and switching among isoforms. Nat Biotechnol 28(5):511-515

Tsai SC, Ames BD (2009) Structural enzymology of polyketide synthases. Methods Enzymol 459:17-47

Valasek A, Kiss ÍÉ, Fodor I, Kovács M, Urbán P, Jámbor É, Cs F, Kerepesi I (2016) Proteomic insight into the primycin fermentation process of Saccharomonospora azurea. Acta Biol Hung 67:424-430

Vályi-Nagy T, Úri J, Szilágyi I (1954) Primycin, a new antibiotic. Nature 174:1105-1106

van Veen H, Konings W (1998) The ABC family of multidrug transporters in microorganisms. Biochim Biophys Acta 1365:31-36

Wang R, Mast Y, Wang J, Zhang W, Zhao G, Wohlleben W, Lu Y, Jiang W (2013) Identification of two-component system AfsQ1/ Q2 regulon and its cross-regulation with GlnR in Streptomyces coelicolor. Mol Microbiol 87(1):30-48

Wang H, Fewer DP, Holm L, Rouhiainen L, Sivonen K (2014) Atlas of nonribosomal peptide and polyketide biosynthetic pathways reveals common occurrence of nonmodular enzymes. Proc Natl Acad Sci USA 111(25):9259-9264

Yadav G, Gokhale RS, Mohanty D (2003) Computational approach for prediction of domain organization and substrate specificity of modular polyketide synthases. J Mol Biol 328:335-363

Yu L, Pan Y, Liu G (2016) A regulatory Gene SCO2140 is involved in antibiotic production and morphological differentiation of Streptomyces coelicolor A3(2). Curr Microbiol 73(2):1-6

Yuan G, Xu L, Xu X, Li P, Zhong Q, Xia H, Hu Y, Li P, Song X, Li J, Liu Q (2019) Azalomycin F5a, a polyhydroxy macrolide binding to the polar head of phospholipid and targeting to lipoteichoic acid to killmethicillin-resistantStaphylococcus aureus. Biomed Pharmacother 109:1940-1950

Zhang F, Shi T, Ji H, Ali I, Huang S, Deng Z, Min Q, Bai L, Zhao Y, Zheng J (2019) Structural Insights into the substrate specificity of acyltransferases from salinomycin polyketide synthase. Biochemistry 58(27):2978-2986

Zschiedrich C, Keidel V, Szurmant H (2016) Molecular mechanisms of two-component signal transduction. J Mol Biol 428(19):3752-3775

Publisher's Note Springer Nature remains neutral with regard to jurisdictional claims in published maps and institutional affiliations. 